\title{
ON STABILITY OF THE CAUCHY FUNCTIONAL EQUATION IN GROUPOIDS
}

\author{
Imke Toborg, Peter Volkmann
}

\begin{abstract}
We give some stability results for the functional equation $a(x y)=$ $a(x)+a(y)$, where $a: G \rightarrow E, G$ being a groupoid and $E$ a Banach space.
\end{abstract}

\section{Introduction}

Let $G$ be a groupoid, i.e., $G$ is a set and for all $x, y \in G$ we have a product $x y \in G$. Furthermore, let $E$ be a Banach space; by $\theta$ we denote its zero element.

We consider the Cauchy equation

$$
a(x y)=a(x)+a(y), \quad x, y \in G,
$$

for functions $a: G \rightarrow E$; its solutions are called additive functions.

A subset $V$ of $E$ is ideally convex (Evgenij Arkad'evič Lifšic [4]), if for every bounded sequence $d_{1}, d_{2}, d_{3}, \ldots$ in $V$ and for every numerical sequence $\alpha_{1}, \alpha_{2}, \alpha_{3}, \ldots \geq 0$ such that $\sum_{k=1}^{\infty} \alpha_{k}=1$ we get $\sum_{k=1}^{\infty} \alpha_{k} d_{k} \in V$.

Let us mention that a convex subset of $E$ is ideally convex, provided it is closed, open or finite dimensional (cf. also Jacek Tabor [8], where the relation between ideally convex sets and stability of the Cauchy equation has been examined; for this relation see also Volkmann [11]). Thus closed and open balls

Received: 01.06.2016. Accepted: 20.09.2016. Published online: 18.12.2016.

(2010) Mathematics Subject Classification: 20N02, 39B82.

Key words and phrases: Cauchy equation, stability, groupoids, Tabor groupoids. 
in $E$ are ideally convex. We denote them by $S(p ; \rho)$ and $S^{0}(p ; \rho)$, respectively ( $p \in E$ being the centre and $\rho \geq 0$ the radius).

Now we consider triplets $(G, E, V)$, where $G, E, V$ essentially are as described before. More precisely, we introduce the following hypothesis:

(H) $G$ is a groupoid, $E$ a Banach space and $V$ a bounded ideally convex subset of $E$.

Definition 1. For a triplet $(G, E, V)$ according to $(\mathrm{H})$ we say it has property $(\mathrm{U})$, if for every $f: G \rightarrow E$ satisfying

$$
f(x y)-f(x)-f(y) \in V, \quad x, y \in G,
$$

there is an additive $a: G \rightarrow E$ such that

$$
a(x)-f(x) \in V, \quad x \in G .
$$

Remark 1. Concerning the special case $V=S(\theta ; \varepsilon)$ (where $\varepsilon>0$ ) we have:

Conditions (2), (3) can be written as

$$
\begin{array}{r}
\|f(x y)-f(x)-f(y)\| \leq \varepsilon, \quad x, y \in G, \\
\|a(x)-f(x)\| \leq \varepsilon, \quad x, y \in G,
\end{array}
$$

respectively, and (U) implies the Hyers-Ulam stability of the Cauchy equation (1) (in the sense of Zenon Moszner [5, Definition 1]; in fact, (U) is equivalent to the Hyers-Ulam stability of (1), which can be seen by using the Theorem 1 below). Finally property (U) for one $\varepsilon>0$ implies already (U) for all $\varepsilon>0$.

Definition 2. For $x \in G, G$ being a groupoid, and $k=0,1,2, \ldots$, the powers $x^{2^{k}}$ are recursively defined by

$$
x^{2^{0}}=x^{1}=x, \quad x^{2^{k+1}}=x^{2^{k}} x^{2^{k}} .
$$

The following result is a stability theorem for the functional equation $h\left(x^{2}\right)=2 h(x)$; see Volkmann [12] for the proof.

TheOrem 1. Consider $(G, E, V)$ according to $(\mathrm{H})$ and let $f: G \rightarrow E$ satisfy

$$
f\left(x^{2}\right)-2 f(x) \in V, \quad x \in G .
$$


Then there is exactly one $h: G \rightarrow E$ such that

$$
h\left(x^{2}\right)=2 h(x), h(x)-f(x) \in V, \quad x \in G,
$$

namely

$$
h(x)=\lim _{n \rightarrow \infty} \frac{1}{2^{n}} f\left(x^{2^{n}}\right), \quad x \in G .
$$

In the next section we use this theorem for a characterization of property (U) and we give some applications. The third section will be devoted to direct products of groupoids and the last one to some concluding remarks.

\section{A characterization of (U) and some consequences}

The following result gives a characterization of property (U).

Theorem 2. Consider $(G, E, V)$ according to $(\mathrm{H})$ and let $f: G \rightarrow E$ satisfy (2). Then the following assertions are equivalent:

(A) There is an additive $a: G \rightarrow E$ satisfying (3).

(B) The function $h: G \rightarrow E$ (given by (6)) is additive.

Proof. (2) implies (4), and therefore we can apply Theorem 1: There is exactly one $h: G \rightarrow E$ satisfying (5), and this function is given by (6).

$(\mathrm{A}) \Rightarrow(\mathrm{B})$ : If $(\mathrm{A})$ holds, then the additive function $a$ has all the properties of $h$, which are stated in (5). The uniqueness of $h$ gives $h=a$, and this proves (B).

(B) $\Rightarrow(\mathrm{A})$ : If $h$ is additive, then $a=h$ obviously leads to (A).

REMARK 2. If the assertions (A), (B) of Theorem 2 are true, then $a=h$.

Theorem 3. Consider $(G, E, V)$ according to $(\mathrm{H})$.

I) If (U) holds for $(G, E, S(\theta ; \varepsilon))(\varepsilon>0)$, then (U) also holds for $(G, E, V)$.

II) If Int $V \neq \emptyset$ and (U) holds for $(G, E, V)$, then (U) also holds for the triplet $(G, E, S(\theta ; \varepsilon))(\varepsilon>0)$.

Proof. I) Let $f: G \rightarrow E$ satisfy (2). We choose $\varepsilon>0$ such that $V \subseteq$ $S(\theta ; \varepsilon)$, and we get

$$
f(x y)-f(x)-f(y) \in S(\theta ; \varepsilon), \quad x, y \in G .
$$


Since (U) holds for $(G, E, S(\theta ; \varepsilon))$, we can apply Theorem 2 with $V$ replaced by $S(\theta ; \varepsilon)$ to get the additivity of $h: G \rightarrow E$ given by $(6)$. This finishes the proof of (U) for $(G, E, V)$ (because of $(\mathrm{B}) \Rightarrow(\mathrm{A})$ from Theorem 2 ).

II) We choose $p \in E$ and $\varepsilon>0$ such that

$$
S(p ; \varepsilon)=p+S(\theta ; \varepsilon) \subseteq V
$$

according to Remark 1 it is sufficient to keep the $\varepsilon$ fixed and to show property $(\mathrm{U})$ for $(G, E, S(\theta ; \varepsilon))$. So let $f: G \rightarrow E$ satisfy $(7)$. For

$$
g(x):=f(x)-p, \quad x \in G,
$$

we have

$$
g(x y)-g(x)-g(y)=f(x y)-f(x)-f(y)+p \in S(p ; \varepsilon) \subseteq V, \quad x, y \in G .
$$

When using (U) for $(G, E, V)$, we get by Theorem 2 the additivity of

$$
k(x)=\lim _{n \rightarrow \infty} \frac{1}{2^{n}} g\left(x^{2^{n}}\right), \quad x \in G .
$$

For $h: G \rightarrow E$ given by (6) we now have

$$
h(x)=\lim _{n \rightarrow \infty} \frac{1}{2^{n}} f\left(x^{2^{n}}\right)=\lim _{n \rightarrow \infty} \frac{1}{2^{n}}\left[g\left(x^{2^{n}}\right)+p\right]=k(x),
$$

hence $h$ is an additive function, and from Theorem 2 we get property (U) for $(G, E, S(\theta ; \varepsilon))$.

The next definition is taken from Roman Badora, Barbara Przebieracz, Volkmann [1]; we adopt the notation $\mathbb{N}=\{0,1,2, \ldots\}, \mathbb{N}^{*}=\mathbb{N} \backslash\{0\}$.

Definition 3. A groupoid $G$ is called Tabor groupoid, if for $x, y \in G$ there exists $k \in \mathbb{N}^{*}$ such that

$$
(x y)^{2^{k}}=x^{2^{k}} y^{2^{k}}
$$

Groups satisfying this condition had been considered by Józef Tabor [9]; we call them Tabor groups. The special case $k=1$ in (8), i.e.,

$$
(x y)^{2}=x^{2} y^{2}, \quad x, y \in G,
$$

had been called square-symmetry by Zsolt Páles, Volkmann, R. Duncan Luce [6]. Of course, (9) holds in commutative semigroups. 
The next theorem has two parts: Part I) is from Volkmann [12]; Part II) is similar to a result of Jürg Rätz [7].

Theorem 4. A triplet $(G, E, V)$ according to $(\mathrm{H})$ satisfies $(\mathrm{U})$ in the following two cases:

I) $G$ is a Tabor groupoid.

II) For every $x \in G$ the set $\left\{x, x^{2}, x^{4}, x^{8}, \ldots\right\}$ is finite.

Proof. Let $f: G \rightarrow E$ satisfy (2). According to Theorem 2 it is sufficient to show the additivity of $h: G \rightarrow E$ (given by (60). In Case I) this can be done by the procedure of Józef Tabor [9]. In Case II) we simply get $h(x) \equiv \theta$.

Remark 3. For commutative semigroups $G$, Part I) goes back to Jacek Tabor [8].

Remark 4. Condition II) is equivalent to the following: For every $x \in G$ there are $m, n \in \mathbb{N}, m \neq n$ such that $x^{2^{m}}=x^{2^{n}}$.

Remark 5. In groupoids $G$, Rätz [7] uses the "left" powers, here for $x \in G$ written as $x^{(n)}:=x\left(x^{(n-1)}\right)(n \in \mathbb{N} \backslash\{0,1\})$, where $x^{(1)}:=x$. By Rätz' Theorem 2, a triplet $(G, E, V)$ according to $(\mathrm{H})$ also satisfies $(\mathrm{U})$ in the following case:

III) For every $x \in G$ the set $\left\{x^{(1)}, x^{(2)}, x^{(3)}, \ldots\right\}$ is finite.

Let us give an example of a groupoid $G$, where I), II) hold but III) does not hold. We take $G=\mathbb{N}$, equipped with the product

$$
x \circ y= \begin{cases}0 & \text { if } x=y, \\ x+y+1 & \text { if } x \neq y, \quad x, y \in \mathbb{N} .\end{cases}
$$

I), II) are obviously satisfied, but

$$
\begin{aligned}
& 1^{(2)}=1 \circ 1=0, \quad 1^{(3)}=1 \circ 0=2, \quad 1^{(4)}=1 \circ 2=4, \\
& 1^{(n)}=2(n-2), \quad n \geq 2 .
\end{aligned}
$$

$\left\{1^{(1)}, 1^{(2)}, 1^{(3)}, \ldots\right\}$ is an infinite set, hence III) does not hold. 


\section{Direct products of groupoids}

Let $G, H$ be groupoids. By the direct product of them we understand (as usual) $G \times H$ equipped with the coordinate-wise defined product, i.e.,

$$
(x, y)(\bar{x}, \bar{y})=(x \bar{x}, y \bar{y}), \quad x, \bar{x} \in G ; y, \bar{y} \in H .
$$

A basic question is the following: Let furthermore $V$ be an ideally convex set in a Banach space $E$, and suppose $(G, E, V),(H, E, V)$ to have property (U). Under which conditions is it true that $(G \times H, E, V)$ also has property (U)?

Concerning this question, Badora, Przebieracz, Volkmann [2] observed that $G \times H$ is a Tabor groupoid, provided $G, H$ have this property and in at least one of them the square-symmetry $(9)$ holds. This fact also follows from Theorem 5 below, which gives a necessary and sufficient condition for $G \times H$ to be a Tabor groupoid.

Definition 4. For groupoids $G$ and $x, y \in G$ we set

$$
T_{G}(x, y)=\left\{k \mid k \in \mathbb{N}^{*},(x y)^{2^{k}}=x^{2^{k}} y^{2^{k}}\right\} .
$$

Remark 6. A groupoid $G$ is a Tabor groupoid if and only if

$$
T_{G}(x, y) \neq \emptyset, \quad x, y \in G,
$$

and in square-symmetric groupoids $G$ we have

$$
T_{G}=\mathbb{N}^{*}, \quad x, y \in G .
$$

TheOREM 5. Let $G, H$ be groupoids. Then $G \times H$ is a Tabor groupoid if and only if $T_{G}(x, y) \cap T_{H}(a, b) \neq \emptyset(x, y \in G ; a, b \in H)$. In particular $G$ and $H$ are Tabor groupoids in this case.

Proof. Consider $x, y \in G$ and $a, b \in H$. The theorem follows from the formula

$$
T_{G \times H}((x, a),(y, b))=T_{G}(x, y) \cap T_{H}(a, b),
$$

which is easily shown: For $k \in T_{G \times H}((x, a),(y, b))$ we have

$$
\begin{aligned}
\left((x y)^{2^{k}},(a b)^{2^{k}}\right) & =(x y, a b)^{2^{k}}=((x, a)(y, b))^{2^{k}} \\
& =(x, a)^{2^{k}}(y, b)^{2^{k}}=\left(x^{2^{k}}, a^{2^{k}}\right)\left(y^{2^{k}}, b^{2^{k}}\right)=\left(x^{2^{k}} y^{2^{k}}, a^{2^{k}} b^{2^{k}}\right),
\end{aligned}
$$


hence $k \in T_{G}(x, y) \cap T_{H}(a, b)$. On the other hand, for $k \in T_{G}(x, y) \cap T_{H}(a, b)$, we get

$$
\begin{aligned}
((x, a)(y, b))^{2^{k}} & =(x y, a b)^{2^{k}}=\left((x y)^{2^{k}},(a b)^{2^{k}}\right)= \\
& =\left(x^{2^{k}} y^{2^{k}}, a^{2^{k}} b^{2^{k}}\right)=\left(x^{2^{k}}, a^{2^{k}}\right)\left(y^{2^{k}}, b^{2^{k}}\right)=(x, a)^{2^{k}}(y, b)^{2^{k}},
\end{aligned}
$$

hence $k \in T_{G \times H}((x, a),(y, b))$.

TheOREm 6. Let $(G, E, V)$ satisfy $(\mathrm{U})$. Let $\Sigma$ be a groupoid with an element $\sigma=\sigma^{2} \in \Sigma$ such that for every $\xi \in \Sigma$ there exists $m \in \mathbb{N}$ yielding $\xi^{2^{m}}=\sigma$. Then $(G \times \Sigma, E, V)$ also has property $(\mathrm{U})$.

Proof. Let $f: G \times \Sigma \rightarrow E$ satisfy

$$
f(x y, \xi \eta)-f(x, \xi)-f(y, \eta) \in V, \quad x, y \in G ; \xi, \eta \in \Sigma .
$$

Then $y=x, \eta=\xi$ leads to

$$
f\left(x^{2}, \xi^{2}\right)-2 f(x, \xi) \in V, \quad x \in G, \xi \in \Sigma,
$$

and Theorem 1 applied to $(G \times \Sigma, E, V)$ shows the existence of exactly one function $h: G \times \Sigma \rightarrow E$ such that

$$
h\left(x^{2}, \xi^{2}\right)=2 h(x, \xi), h(x, \xi)-f(x, \xi) \in V, \quad x \in G, \xi \in \Sigma .
$$

This function is given by

$$
h(x, \xi)=\lim _{n \rightarrow \infty} \frac{1}{2^{n}} f\left(x^{2^{n}}, \xi^{2^{n}}\right), \quad x \in G, \xi \in \Sigma .
$$

The choice $\xi=\eta=\sigma$ in 10 leads to

$$
f(x y, \sigma)-f(x, \sigma)-f(y, \sigma) \in V, \quad x, y \in G,
$$

and since $(G, E, V)$ has the property (U), we get an additive $a: G \rightarrow E$ such that

$$
a(x)-f(x, \sigma) \in V, \quad x \in G .
$$

By Theorem 2 and Remark 2 we now have

$$
a(x)=\lim _{n \rightarrow \infty} \frac{1}{2^{n}} f\left(x^{2^{n}}, \sigma\right)=h(x, \sigma), \quad x \in G .
$$


For $\xi \in \Sigma$ let $m \in \mathbb{N}$ be such that $\xi^{2^{m}}=\sigma$. Then we get by (11), (12) for $x \in G$ :

$$
\begin{aligned}
h(x, \xi) & =\frac{1}{2} h\left(x^{2}, \xi^{2}\right)=\ldots \\
& =\frac{1}{2^{m}} h\left(x^{2^{m}}, \sigma\right)=\frac{1}{2^{m}} a\left(x^{2^{m}}\right)=a(x) .
\end{aligned}
$$

So we have

$$
h(x, \xi)=a(x), \quad x \in G, \xi \in \Sigma,
$$

and therefore the function $h: G \times \Sigma \rightarrow E$ occuring in (11) is additive. Indeed, for $x, y \in G$ and $\xi, \eta \in \Sigma$ we get

$$
\begin{aligned}
h((x, \xi)(y, \eta)) & =h(x y, \xi \eta)=a(x y) \\
& =a(x)+a(y)=h(x, \xi)+h(y, \eta) .
\end{aligned}
$$

This finishes the proof of $(\mathrm{U})$ for $(G \times \Sigma, E, V)$.

REMARK 7. When taking $G=\{0\}$ (a singleton) and observing $\Sigma \cong\{0\} \times$ $\Sigma$, we see that $(\Sigma, E, V)$ has property $(\mathrm{U})$. In fact, $\Sigma$ is a Tabor groupoid (a proof is easy), which in the semigroup-case already is known from Badora, Przebieracz, Volkmann [2, Theorem 3, Case I)].

The next theorem is trivial (we omit the proof), but it may be useful in applications.

Theorem 7. Let $\left(G_{1}, E_{1}, V_{1}\right),\left(G_{2}, E_{2}, V_{2}\right)$ have property $(\mathrm{U})$ and let the function $f: G_{1} \times G_{2} \rightarrow E_{1} \times E_{2}$ be given by

$$
f\left(x_{1}, x_{2}\right)=\left(f_{1}\left(x_{1}\right), f_{2}\left(x_{2}\right)\right), \quad\left(x_{1}, x_{2}\right) \in G_{1} \times G_{2},
$$

where $f_{j}: G_{j} \rightarrow E_{j}(j=1,2)$. Suppose

$$
f(x y)-f(x)-f(y) \in V_{1} \times V_{2}, \quad x, y \in G_{1} \times G_{2} .
$$

Then there exists an additive $a: G_{1} \times G_{2} \rightarrow E_{1} \times E_{2}$ such that

$$
a(x)-f(x) \in V_{1} \times V_{2}, \quad x \in G_{1} \times G_{2} .
$$


REMARK 8. If $E_{1} \times E_{2}$ is normed by $\left\|\left(x_{1}, x_{2}\right)\right\|=\max \left\{\left\|x_{1}\right\|,\left\|x_{2}\right\|\right\}$ and $V_{1}, V_{2}$ are closed (or open) $\varepsilon$-balls centered at $\theta$, then $V_{1} \times V_{2}$ also is a closed (or open) $\varepsilon$-ball centered at $\theta$, i.e.,

$$
\begin{aligned}
& S_{E_{1}}(\theta ; \varepsilon) \times S_{E_{2}}(\theta ; \varepsilon)=S_{E_{1} \times E_{2}}(\theta ; \varepsilon), \\
& S_{E_{1}}^{0}(\theta ; \varepsilon) \times S_{E_{2}}^{0}(\theta ; \varepsilon)=S_{E_{1} \times E_{2}}^{0}(\theta ; \varepsilon) .
\end{aligned}
$$

Of course, this remark concerns in particular the Hyers-Ulam stability mentioned in Remark 1.

Let us conclude this section by recalling some known results for groups.

TheORem 8. Let $G$ be a group.

I) If every element of $G$ has odd order, then $G$ is a Tabor group.

II) If every element of $G$ has an order $2^{n}$ (where $n \in \mathbb{N}$ ), then $G$ is a Tabor group.

III) If $G \cong G_{1} \times G_{2}$ with groups $G_{1}, G_{2}$ as in I), II) (respectively), then $G$ is a Tabor group.

IV) Any finite Tabor group $G$ has the form given in III).

Remark 9. I), II) follow from Badora, Przebieracz, Volkmann [2], concerning II) cf. Remark 7; III), IV) are from Toborg [10].

\section{Final remarks}

Let $F(a, b)$ be the free group with two generators and let $\mathbb{R}$ denote the space of the reals. Gian Luigi Forti [3] has shown that the triplet $(F(a, b), \mathbb{R}$, $[-1,1])$ does not have property $(\mathrm{U})$. Thus $F(a, b)$ is not a Tabor group. Now the question is of interest, whether there exist torsion free non commutative Tabor groups.

Finally let us mention that all groupoids with two elements are Tabor groupoids; this can be easily checked. On the other hand, there is a groupoid $G=\{a, b, c\}$ which is not a Tabor groupoid: It is sufficient to require $a^{2}=$ $a, a b=c, b^{2}=c^{2}=b \neq c$. Indeed, assume for some $k \in \mathbb{N}^{*}$ that

$$
(a b)^{2^{k}}=a^{2^{k}} b^{2^{k}} .
$$

We get $c^{2^{k}}=a b$, hence $b=c$, which is a contradiction. 
Acknowledgement. We cordially thank Mrs. Marion Ewald for her typesetting of this paper.

\section{References}

[1] Badora R., Przebieracz B., Volkmann P., Stability of the Pexider functional equation, Ann. Math. Sil. 24 (2010), 7-13.

[2] Badora R., Przebieracz B., Volkmann P., On Tabor groupoids and stability of some functional equations, Aequationes Math. 87 (2014), 165-171.

[3] Forti G.L., Remark 11 (at the 22nd International Symposium on Functional Equations, Oberwolfach 1984), Aequationes Math. 29 (1985), 90-91.

[4] Lifšic E.A., Ideal'no vypuklye množestva, Funkcional'. Analiz Priložen. 4 (1970), no. $4,76-77$.

[5] Moszner Z., On the stability of functional equations, Aequationes Math. 77 (2009), 33-88.

[6] Páles Z., Volkmann P., Luce R.D., Hyers-Ulam stability of functional equations with a square-symmetric operation, Proc. Nat. Acad. Sci. U.S.A. 95 (1998), 12772-12775.

[7] Rätz J., On approximately additive mappings, in: General inequalities 2, International Series of Numerical Mathematics 47, Birkhäuser, Basel, 1980, pp. 233-251.

[8] Tabor Jacek, Ideally convex sets and Hyers theorem, Funkcial. Ekvac. 43 (2000), 121125.

[9] Tabor Józef, Remark 18 (at the 22nd International Symposium on Functional Equations, Oberwolfach 1984), Aequationes Math. 29 (1985), 96.

[10] Toborg I., Tabor groups with finiteness conditions, Aequationes Math. 90 (2016), 699704.

[11] Volkmann P., Zur Rolle der ideal konvexen Mengen bei der Stabilität der Cauchyschen Funktionalgleichung, Sem. LV, no. 6 (1999), 6 pp., http://www.math.us.edu.pl/smdk

[12] Volkmann P., O stabilności równań funkcyjnych o jednej zmiennej, Sem. LV, no. 11 (2001), 6 pp., Errata ibid. no. 11bis (2003), 1 p., http://www.math.us.edu.pl/smdk

INSTITUT FÜR MATHEMATIK

Naturwissenschaftliche FakUltät II

MARTIN-Luther-UniversitÄT

HALle-WitTEnBerg

06099 Halle (SaAle)

Germany

e-mail: imke.toborg@mathematik.uni-halle.de
INSTITUT FÜR ANALYSIS

KIT

76128 KARLSRUHE

Germany 